\title{
Diagnosis and management of vertigo
}

\author{
GM Halmagyi
}

\begin{abstract}
Vertigo is an illusion of rotation due to a disorder of the vestibular system, almost always peripheral. In the history it must be distinguished from pre-syncope, seizures and panic attacks. A single attack of acute, isolated spontaneous vertigo lasting a day or more is due either to vestibular neuritis or cerebellar infarction; distinguishing between the two requires mastery of the head impulse test. Recurrent vertigo is mostly due to benign paroxysmal positioning vertigo (BPPV), Menière's disease or migraine. With a good history, a positional test, an audiogram and a caloric test, it is usually possible to distinguish between these. BPPV is the single most common cause of recurrent vertigo and can usually be cured immediately with a particle repositioning manoeuvre. Posterior circulation ischaemia very rarely causes isolated vertigo attacks and when it does the attacks are brief and frequent and the history is short.
\end{abstract}

KEY WORDS: benign paroxysmal positioning vertigo (BPPV), cerebellar infarction, labyrinthitis, Menière's disease, migraine, vertigo, vestibular BPV, vestibular neuritis

\section{What vertigo is and is not}

Vertigo is an illusion of rotation caused by asymmetry of neural activity between the left and right vestibular nuclei. This can be induced in anyone

- by simply spinning them at constant speed for about 20 seconds and then suddenly stopping them

- by heating or cooling one labyrinth, as happens in the caloric test

- by otoconial particles (calcium-containing debris: literally 'earstones') moving along semicircular canal duct, as happens in benign paroxysmal positional vertigo (BPPV).

It can also be produced by sudden unilateral destruction of a normal vestibular end-organ, nerve or nucleus or vestibulo-cerebellum, a structure that normally inhibits the ipsilateral vestibular nucleus. (Bilateral simultaneous vestibular destruction does not produce vertigo - in fact, a patient with bilateral vestibular loss will never again experience vertigo from unilateral or asymmetrical vestibular stimulation.)

Vertigo is always temporary. Even after the vestibular nerve on one side has been surgically severed, the resulting vertigo (and nystagmus) will abate within a few days. This is not because the vestibular nerve has re-anastomosed but because profound neurochemical changes occuring in the brainstem during the process of vestibular compensation restore symmetrical vestibular nucleus activity. Vertigo is always made worse by head movement; thus patients who are 'dizzy all the time' and are happy to move around whilst dizzy do not have vertigo.

Patients with aural vertigo should not lose consciousness but some patients cannot give confident and convincing answers to such simple questions as 'Did it feel as if you were losing balance or as if you were losing consciousness? Did you feel as if you were going to pass out or fall over?' Patients with vertigo might lose consciousness if they have been vomiting a lot, or if they incurred a head injury when they fell.

Witness descriptions are not much help in identifying vertigo, but can be helpful in identifying other paroxysmal disorders such as seizure and syncope. Whereas panic attacks, especially with hyperventilation, commonly cause a sense of dizziness that is not

\section{Key Points}

In a patient with vertigo

Do the positional test and if positive for BPPV go on to a particle repositioning manoeuvre

Do the head impulse test in the patient and if negative think of cerebellar infarction in a patient with a first-ever attack of acute isolated spontaneous vertigo

Order an audiogram and a caloric test and if they are normal think of migraine rather than Menière's disease in a patient with recurrent vertigo

Think of bilateral vestibular loss due to gentamicin, normal pressure hydrocephalus, early cerebellar ataxia, early progressive supranuclear palsy, sensory peripheral neuropathy and orthostatic tremor in the patient who is off balance for no obvious reason

Forget about vertebro-basilar transient ischaemic attacks as a cause of isolated recurrent vertigo 
actually vertigo, patients with recurrent undiagnosed vertigo can develop panic attacks, particularly if the vertigo attacks are reassuringly put down to 'just a little anxiety'. This is one of those unusual situations where patients do as they are told and develop anxiety, panic, and even agoraphobia. ' 'Phobic postural vertigo' is a variant of this problem: patients, often with obsessive-compulsive personalities, complain of a mild subjective disturbance of balance while standing or walking, with momentary illusions of motion. ${ }^{2}$ The symptoms usually occur in specific places or situations, and are associated with a distressing anxiety. Many cases follow a well-documented peripheral vestibulopathy. While not everyone likes the name 'phobic postural vertigo,', ${ }^{3}$ typically such patients do well with simple non-judgemental support and an accurate non-patronising explanation. ${ }^{4}$

\section{Benign paroxysmal positioning vertigo (BPPV)}

Benign paroxysmal positioning vertigo (BPPV) is the single most common cause of vertigo and many patients give a story that is clear enough for a telephone diagnosis. 'Doctor, whenever I turn in bed at night, or I hang the washing on the line or look under my car...'; they don't even have to say that they are dizzy. Mostly BPPV will occur in bouts lasting several weeks and will then spontaneously remit, only to return weeks, months, or even years later. Those with repeated bouts of vertigo over several decades, with no abnormalities on examination, have BPPV. The cause of BPPV is the movement of stray otoconial particles within the duct of one semicircular canal, usually the posterior.

The positioning test, as described by Bárány, perfected by Dix and popularised by Hallpike, is the cornerstone of diagnosis and now of treatment. ${ }^{5}$ The idea of the test is to make any otoconia in the posterior semicircular canal move and so provoke vertigo and nystagmus. Eventually Brandt and Daroff, and then Semont et $a l^{6}$ and Epley ${ }^{7}$ realised that making the otoconia move within the duct also allowed them to be removed from the duct, which also means that if one cannot provoke the BPPV one cannot cure it.

Consider a patient with left posterior semicircular canal BPPV. Whilst sitting, the posterior semicircular canal is gravitationally vertical, with the ampulla lowermost; any otoconia in the duct will be next to the cupula (Fig 1: START). The patient's head is turned to the left and the patient is rapidly pitched backwards until the head is hanging over the end of the bed (Fig 1: 1). The otoconia will now fall down from the cupula and come to rest at the midpoint of the duct. As they fall away from the cupula they create a negative fluid pressure that pulls on the cupula, so exciting primary afferents of the posterior semicircular canal and producing a brief paroxysm of vertigo (20 seconds) and nystagmus; upbeating and counterclockwise (from the patient's point of view) fast phases. ${ }^{8}$ The patient is now slowly rotated through 180 degrees towards his right, until the right side of his face is touching the bed (Fig 1: 2 to 6). The posterior semicircular canal will have been inverted and the otoconia should move further along the semicircular canal duct. A further, less severe, paroxysm of vertigo and counterclockwise upbeating nystagmus results. The patient, still face down, now stands up and the otoconia will continue back into the vestibule (Fig 1: FINISH). This is the particle repositioning or 'liberatory' manoeuvre as described by Epley. It stops BPPV attacks in four out of five patients. ${ }^{11}$ Those resistant to repeated repositioning manoeuvres can be cured by surgical occlusion of the posterior semicircular canal. ${ }^{9}$ Post-traumatic cases in particular can be bilateral but it is sometimes difficult to tell bilateral BPPV from unilateral BPPV. ${ }^{10}$

In most patients with BPPV, there are no other symptoms and there is no demonstrable abnormality of vestibular or auditory function. In a few, it follows acute vestibular neuritis or occurs during the course of a progressive inner ear disease (for example, Menière's disease, Cogan's syndrome). Very rarely is a clinical picture identical to typical BPPV produced by a
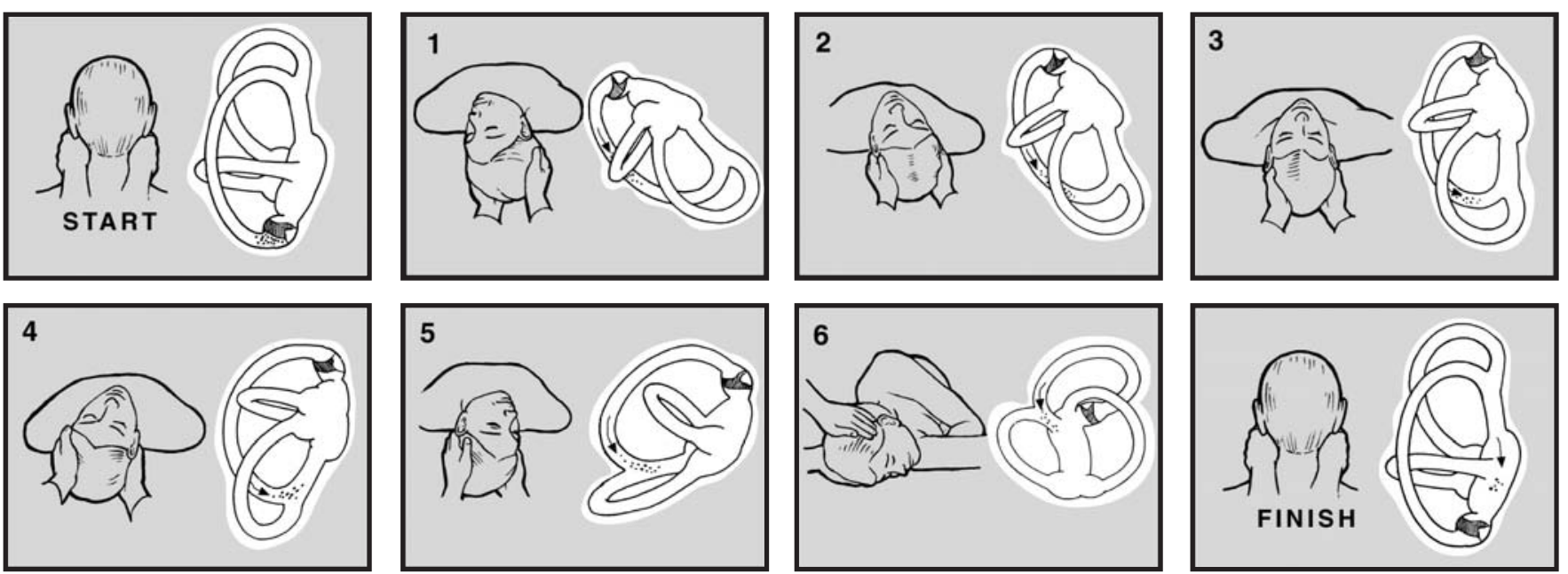

Fig 1. Left posterior semicircular canal BPPV. From an upright sitting position (START) the patient is rapidly placed in the left Hallpike position (1). The head is then slowly rotated (2-5) to the right. The patient then turns to his right side and the head is turned by another 90 degrees to the right (6). After two to three minutes the patient slowly sits up again (FINISH). 
posterior fossa problem such as a tumour, malformation, or degeneration, and BPPV is common enough for a patient to have both.

Lateral semicircular canal BPPV is a variant in which the nystagmus is horizontal and usually beats toward the lowermost ear indicating that the otoconia in the duct are falling toward the cupula. ${ }^{11}$ Treatment of lateral semicircular canal BPPV is less reliable than that of semicircular posterior canal BPPV and largely consists of rotating the recumbent patient 360 degrees from the bad side towards the good side and then having the patient sleep only on the good side so that the otoconia can find their way out of the lateral semicircular canal back into the vestibule. ${ }^{12}$

\section{Recurrent spontaneous vertigo: is it Menière's disease or migraine?}

The patient with repeated attacks of spontaneous vertigo each lasting an hour or more has either Menière's disease or migraine. The presumed pathophysiological basis of Menière's disease is episodic endolymphatic hypertension which produces devastating attacks of spontaneous vertigo with nausea and vomiting, together with a low frequency hearing loss, a low frequency tinnitus, and a sense of fullness or blockage in the affected ear. ${ }^{13,14}$ The vertigo attacks usually last for a few hours, but the tinnitus and hearing loss might continue for days. The attacks might occur days, months, or even years apart. After the first few attacks of vertigo, vestibular and cochlear function recover, so caloric testing and pure tone audiogram will both be normal. Later, after many more attacks of vertigo, a permanent loss of auditory and of vestibular function becomes apparent even between attacks. Menière's disease can remit permanently at any stage, but if it does progress, in the late stages the patient is still subject to attacks of spontaneous vertigo with the added misery of continual tinnitus in a deaf ear that distorts and recruits ('Speak up.' 'There is no need to shout!') Strict dietary sodium restriction aiming for a urinary sodium less than $50 \mathrm{mmol} /$ day is the most effective medical therapy (there has been no proper clinical trial), and is more effective and less troublesome than diuretics. Surgery can stop the vertigo attacks but cannot restore the hearing. Endolymphatic sac surgery of various sorts is becoming less popular, is sometimes effective, and should not destroy any auditory or vestibular function. A surgical, or more simply, a transtympanic gentamicin labyrinthectomy will stop the vertigo but will not save the hearing, whereas an intracranial vestibular nerve section will also stop the vertigo attacks and save whatever hearing remains.

A common clinical problem is the patient who presents with repeated attacks of spontaneous vertigo, unaware of any temporary deafness, tinnitus, or fullness in one ear at the time of a vertigo attack, with no clinical abnormalities, a normal audiogram, and a normal caloric test. (It is not easy to evaluate a dizzy patient without access to reliable, reproducible caloric testing.) Could this patient have Menière's disease? The answer is, of course, yes. The patient might in fact have had a temporary low frequency hearing loss during the vertigo attack but would not have noticed it. Patients often do not notice a slight hearing loss in one ear, particularly if the loss is mainly below $1.5 \mathrm{kHz}$, the centre of the speech spectrum, and during an attack of vertigo and vomiting when they are too busy being dizzy to worry about a little deafness in one ear. There are also patients who have repeated spontaneous vertigo attacks for many years before they develop a unilateral tinnitus and hearing loss so that the diagnosis of Menière's disease finally becomes obvious. But on the other hand, such a patient could also have vestibular migraine.

Those who have migraine headaches have more vertigo than those who do not. ${ }^{15}$ Some migraineurs will sometimes have vertigo as their migrainous aura, and will then go on to develop a typical hemicranial headache with nausea and vomiting. Attacks such as these have been called basilar migraine, although Bickerstaff ${ }^{16}$ probably meant something much more dramatic when he coined the phrase. Other migraineurs will have repeated attacks of spontaneous vertigo, apart from the attacks of headache, typically lasting less than an hour, with nausea and even vomiting, but without any hearing disturbance or headache at the time. ${ }^{17}$ The potential mechanism by which migraine might produce vertigo attacks is as open and as contentious as the mechanism of migraine itself. Nevertheless, vertigo attacks in migraineurs often respond to medications used to treat migraine headaches such as an ergot, a triptan, or even aspirin. In some patients, the vertigo attacks can be prevented by regular treatment with a beta-blocker, a calcium channel blocker, a tricyclic, valproate, acetazolamide, or methysergide. ${ }^{18}$ As transient neurological events of obscure origin, in the absence of migraine headaches, are common enough in young people, ${ }^{19}$ it is likely that the same mechanism, whether it is migrainous or not, can also cause vertigo attacks in patients who have never had migraine headaches.

\section{The patient with a first attack of acute spontaneous vertigo}

This is the patient who suddenly develops, for the first time ever, such intense spontaneous vertigo, nausea, and vomiting that she or he ends up in a hospital emergency department. There the house doctors will guess, usually correctly, that the patient has 'labyrinthitis', although some will call it a 'middle ear infection' and prescribe an antibiotic. Few, however, will be able to elicit the essential clinical signs of labyrinthitis, which is regrettable as these are simple, and necessary to distinguish labyrinthitis from cerebellar infarction without magnetic resonance imaging (MRI).

\section{Acute vestibular neuritis}

Sudden, spontaneous, isolated, unilateral, total, or subtotal loss of peripheral vestibular function is a common and dramatic event. It is usually ascribed to viral infection or to a para-infectious event, and has been called 'labyrinthitis', 'vestibular neuritis', 'vestibular neuronitis', and 'neurolabyrinthitis'. The evidence for viral infection is thin and some prefer to call it 'acute unilateral peripheral vestibulopathy. ${ }^{20}$

In patients with combined superior and inferior vestibular 
neuritis, the clinical signs are the same as those that occur after a labyrinthectomy or a vestibular neurectomy. There is a horizontal-torsional spontaneous nystagmus with the slow phases towards the affected ear - that is, quick phases towards the unaffected ear. The nystagmus is always strictly unidirectional - bidirectional gaze-evoked nystagmus excludes the diagnosis. The nystagmus is, to some extent, always suppressed by visual fixation, and for that reason it might be missed on the standard clinical examination. When some means are used to view the eyes in the absence of visual fixation, such as ophthalmoscopy with the other eye covered, ${ }^{21}$ or Frenzel glasses, the nystagmus will be evident. Ophthalmoscopy with the other eye covered allows the clinician to see fundus nystagmus, released from fixation suppression since the viewed eye is blinded by the ophthalmoscope light and the other eye by the covering hand. Frenzel glasses use +30 dioptre lenses to blur the patient's vision while allowing the clinician to see the patient's eyes. Infrared video goggles have largely replaced optical Frenzel glasses.

The head impulse test (Fig 2) is invariably positive and shows absent lateral semicircular canal function on the affected side. ${ }^{22}$ The patient, although unsteady, can stand without support with the eyes open but rotates toward the side of the lesion when trying to march on the spot with the eyes closed - a positive Fukuda or Unterberger test to the cognoscenti. There is an ocular tilt reaction, always toward the affected side, but this is rarely obvious clinically: there might be a head tilt toward the affected side and sometimes a vertical diplopia, with the higher image coming from the eye on the side of the affected ear. However, the cardinal sign of the ocular tilt reaction, a conjugate torsional offset of the eyeballs toward the affected side, can only be seen with indirect ophthalmoscopy or with fundus photography. Nevertheless, it can be inferred by testing the subjective visual horizontal, an easy test that can be done in any clinical neurophysiology department. ${ }^{23}$ In some patients the disorder only affects the superior vestibular nerve and spares the inferior division of the vestibular nerve $\mathrm{e}^{24}$ so that the patient with vestibular neuritis is able to develop BPPV, presumably as a consequence of otoconia being shed from the utricle into the duct of the posterior semicircular canal.

\section{Cerebellar infarction}

The main differential diagnosis of acute vestibular neuritis is cerebellar infarction. There are several ways to tell the difference clinically. Firstly, the head impulse test can be used. In the clinical context of a first ever attack of acute spontaneous vertigo, if the head impulse test is positive then the patient has acute vestibular neuritis and if the head impulse test is negative, then the patient definitely does not have acute vestibular neuritis affecting the superior vestibular nerve, and might have a cerebellar infarct. Secondly, with a cerebellar infarct the nystagmus might be bilateral, might be vertical, and will not be well
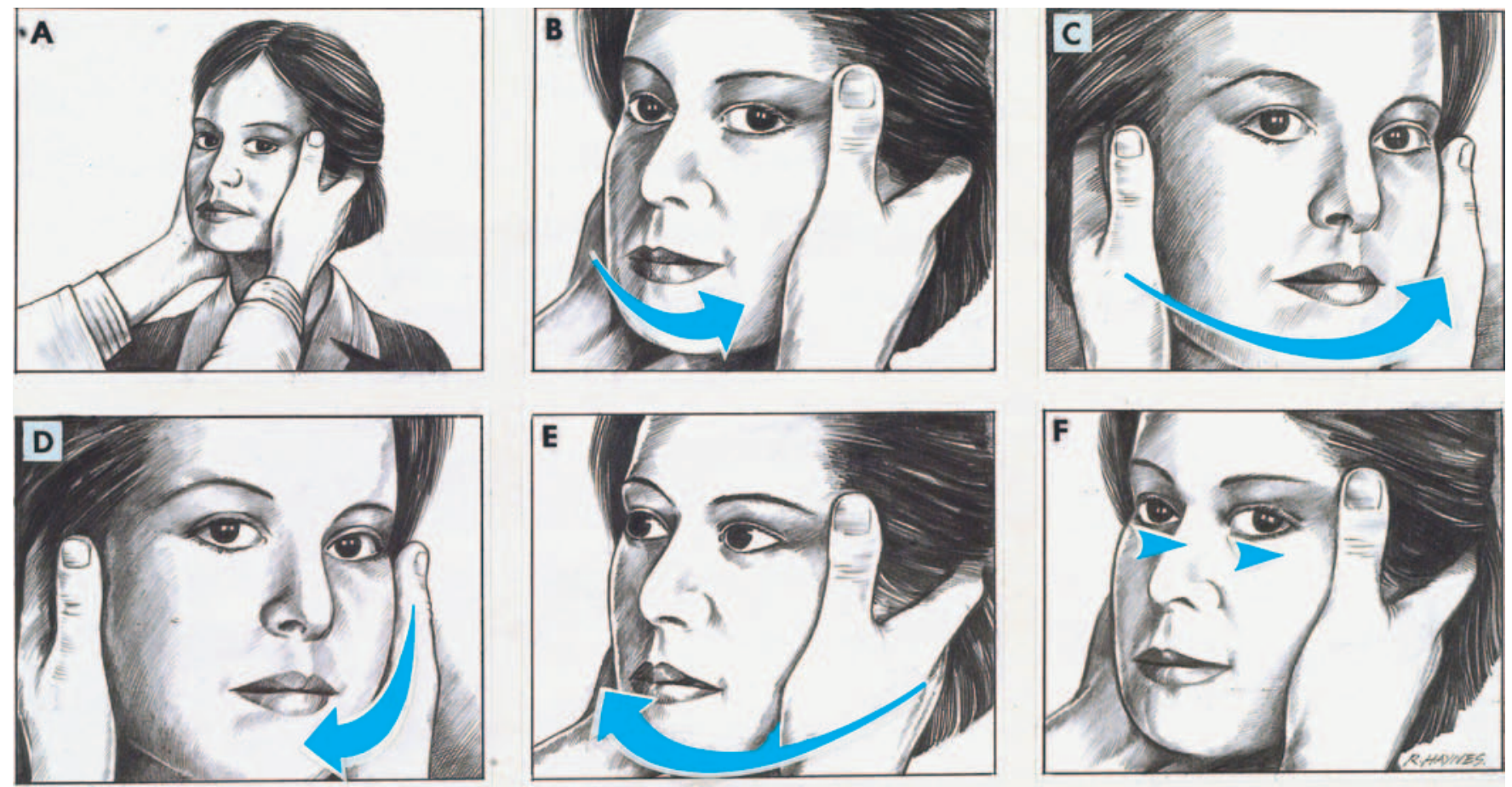

Fig 2. The head impulse test. The examiner turns the patient's head as rapidly as possible about 15 degrees to one side and observes the ability of the patient to keep fixating on a distant target. The patient illustrated has a right peripheral vestibular lesion with a severe loss of right lateral semicircular canal function. While the examiner turns the patient's head towards the normal left side (top row) the patient is able to keep fixating on target. By contrast, when the examiner turns the patient's head to the right, the vestibulo-ocular reflex fails and the patient cannot keep fixating on target $(E)$ so that she needs to make a voluntary rapid eye movement - ie a saccade, back to target $(F)$ after the head impulse has finished; this can be easily observed by the examiner. It is essential that the head is turned as rapidly as possible otherwise smooth pursuit eye movements will compensate for the head turn. 
suppressed by visual fixation - that is, it will be obvious even without Frenzel glasses. Thirdly, a patient with a cerebellar infarct usually cannot stand without support even with the eyes open, whereas the patient with acute vestibular neuritis usually can. Unfamiliarity with the head impulse test is the most common reason that a confident clinical diagnosis of acute vestibular neuritis cannot be made and in that case imaging will be required, and as computed tomography (CT) misses many acute cerebellar infarcts, this means MRI.

Cerebellar infarction is worth diagnosing, firstly because about a third of cases will develop acute, potentially lethal, posterior fossa brain oedema requiring emergency neurosurgical decompression, and secondly because most cases are due to cardiogenic embolism and will require long-term oral anticoagulation to prevent recurrences. ${ }^{25}$ Although brainstem infarction and brainstem multiple sclerosis might produce an attack with predominantly vertigo and nystagmus, particularly if the plaque involves the 8 th nerve root entry zone, ${ }^{26}$ there will generally be other, albeit subtle signs to indicate that the process is in the brainstem and not in the labyrinth.

\section{Late complications of vestibular neuritis}

The patient who has an attack of acute spontaneous vertigo and is seen at home by his general practitioner and treated as having vestibular neuritis, will only be sent to a consultant if complications occur. So if the patient has actually had vestibular neuritis, there is a 1 in 5 chance that he will present later with attacks of typical posterior semicircular canal BPPV or with imbalance due to inadequate vestibular function. If the patient has had a small embolic infarct in the cerebellum, he might not present until he has had another one, this time perhaps not in the cerebellum but elsewhere.

\section{The patient who is off balance}

There are many reasons for a patient to be off balance while standing or walking, a symptom that many patients will call 'dizziness'. Although it is true that the older the patient the less likely that a single diagnosis can account for the problem, there are some diagnoses that tend to be forgotten.

\section{Bilateral vestibulopathy}

Bilateral vestibular loss causes ataxia and oscillopsia, not vertigo, ${ }^{27}$ and in the absence of any significant and relevant hearing loss it can cause diagnostic difficulties because an aural cause might not be considered in the differential diagnosis of imbalance. The patient will be able to walk perfectly well heel to toe and the only easily demonstrable abnormality will be an inability to stand, with the eyes closed, but only when trying to do so on a soft, yielding surface such as a mattress or two pillows - a sort of Romberg's test. The head impulse test will be positive to the left, right, up, and down, and caloric and rotational tests will show bilaterally absent or severely impaired lateral semicircular canal vestibulo-ocular reflexes. Sometimes patients with severe unilateral loss of vestibular function will present with the same symptoms. ${ }^{28}$ The most common known cause of bilateral vestibular loss without hearing loss is gentamicin toxicity. ${ }^{29}$ Systemic gentamicin is not cochleotoxic in humans - it does not cause deafness or tinnitus, but as far as the vestibular system is concerned there is no safe dose, and any patient who notices imbalance after a hospital admission has gentamicin vestibulotoxicity until proved otherwise. As the patient might not be aware of having been given gentamicin, it might be necessary to requisition the hospital's records.

\section{Hydrocephalus}

Patients with normal pressure hydrocephalus can present with imbalance rather than incontinence or memory loss and it is easy enough to discount ventriculomegaly in the presence of cortical atrophy. In these patients, gait can improve after cerebrospinal fluid shunting. ${ }^{30,31}$

\section{Posterior fossa lesions}

Posterior fossa tumours or, particularly those within the fourth ventricle, prepontine or cerebellopontine cisterns ${ }^{32}$ and Chiari malformations, ${ }^{33}$ are still a trap if the patient has only had CT imaging. The sole complaint might be of a vague imbalance and perhaps some positional vertigo with no clinical abnormalities. This type of presentation deserves MRI. 


\section{Progressive supranuclear palsy}

This rare extrapyramidal disorder can present with imbalance and a tendency to fall, well before any eye signs have developed. ${ }^{34}$ The only clue might be some axial rigidity and a somewhat impassive countenance.

\section{Cerebellar ataxia}

A mild cerebellar gait ataxia with no appendicular ataxia, no nystagmus or other abnormal eye signs can be the presenting feature of a late onset dominantly inherited spinocerebellar ataxia, a paraneoplastic cerebellar degeneration or multiple system atrophy. Genetic testing, ${ }^{35}$ serum anti-neuronal antibodies, whole body CT or positron emission tomography (PET) scan, ${ }^{36}$ tilttesting, brain MRI and anal sphincter electromyography ${ }^{37}$ can help diagnose these diseases.

\section{Orthostatic tremor}

This disorder typically causes no problem with walking but does cause a feeling of imbalance while standing. The patient might not have noticed the fine high frequency tremor, which will be obvious with surface electronmyography (EMG), ${ }^{38}$ and can even be heard with a stethoscope over the quadriceps or tibialis anterior. $^{39}$

\section{Spinal cord disease}

Patients with cervical or thoracic myelopathy, usually compressive from spondylosis or meningioma, can present with imbalance and remarkably little in the way of pyramidal signs or sensory deficits in the lower limbs. As well as MRI, a serum B12 needs to be done.

\section{Sensory neuropathy}

While most patients with large fibre peripheral neuropathy bad enough to make them off balance will have distal paraesthesiae, sensory impairment and loss of ankle jerks, the non-specific loss of balance might be out of proportion to the specific signs of peripheral neuropathy. ${ }^{40}$ Nerve conduction studies will in these cases be quite abnormal.

\section{Diagnoses that are likely to be wrong in a patient with vertigo}

Certain diagnoses should not really be made in a patient who has repeated isolated vertigo attacks, a normal clinical examination and no objective loss of auditory or vestibular function. Acute otitis media does not cause vertigo unless there is a suppurative labyrinthitis. Chronic otitis media can, rarely, produce vertigo due secondary endolymphatic hydrops or cholesteatoma causing a perilymph fistula but not without a hearing loss. Probably all cases of 'spontaneous' perilymph fistula are due to a dehiscence of the superior semicircular canal into the middle cranial fossa ${ }^{41,42}$ rather than of the lateral semicircular canal into the middle ear. Acoustic neuroma (more correctly vestibular schwannoma) rarely produces attacks of spontaneous vertigo, and maybe never in a patient who has no fixed unilateral or asymmetric abnormalities of auditory or vestibular function. ${ }^{43}$ Microvascular loop compression is a validated cause of paroxysmal symptoms related to the trigeminal and facial nerves, but the evidence that microvascular compression of the vestibular nerve causes paroxysmal vestibular symptoms, or any symptoms at all, is unconvincing. ${ }^{44}$ The anterior inferior cerebellar artery normally loops into the internal auditory canal and is not a bona fide cause of symptoms. Although frequent brief attacks of vertigo accompanied by unilateral hyperacusis or tinnitus can respond to treatment with carbamazepine and have been called 'vestibular paroxysmia', ${ }^{45}$ there is scant evidence for symptomatic microvascular compression in these patients.

Transient vertebrobasilar ischaemia is a difficult diagnosis and unlikely to be correct in a patient who has attacks consisting only of vertigo. ${ }^{46}$ Nonetheless, it could be suspected in a patient in whom only some of the vertigo attacks are accompanied by other symptoms of brainstem dysfunction such as diplopia. ${ }^{47-49}$ In the absence of any simultaneous brainstem symptoms, a short history, of days rather than months, of frequent brief vertigo attacks lasting minutes rather than hours several times a day, should raise the suspicion that the attacks are posterior circulation transient ischaemic attacks (TIAs). Hearing symptoms, tinnitus and deafness, if unilateral and occurring at the same time as the vertigo attacks, suggest an aural rather than a brainstem problem. By contrast, sudden, temporary bilateral hearing loss does suggest brainstem ischaemia. ${ }^{50}$ Vestibular function tests are expected to be normal and are only of help in a negative sense: if they show a definite unilateral abnormality which will suggest an aural rather than a central cause for the vertigo.

\section{Summary}

In the patient with repeated attacks of isolated vertigo:

1 Always do a positional test.

2 Learn to do the particle repositioning manoeuvre.

3 Always order an audiogram.

4 Try migraine treatment.

5 Put vertebrobasilar insufficiency at the bottom of the list.

\section{In the patient having the first ever attack of acute spontaneous vertigo:}

1 Learn to do the head impulse test.

2 Always think of cerebellar infarction.

\section{In the patient who is off balance:}

1 Think of gentamicin vestibulotoxicity.

2 Think of normal pressure hydrocephalus.

3 Beware of the posterior fossa tumour or malformation.

4 Think of orthostatic tremor.

5 Consider spinal cord or peripheral nerve pathology. 


\section{References}

1 Jacob, RG, Furman JM, Durrant JD, Turner SM. Panic, agoraphobia and vestibular dysfunction. Am J Psychiatry 1996;153:503-12.

2 Brandt T. Phobic postural vertigo. Neurology 1996;46:1515-9.

3 Bronstein AM, Gresty MA, Luxon LM, Ron MA et al. Phobic postural vertigo; letter and reply. Neurology 1997;49:1480-1.

4 Kapfhammer HP, Mayer C, Hock U, Huppert D et al. Course of illness in phobic postural vertigo. Acta Neurol Scand 1997;95:23-8.

5 Lanska DJ, Remler B. Benign paroxysmal positioning vertigo: classic descriptions, origins of the provocative technique and conceptual developments. Neurology 1997;48:1167-77.

6 Epley J. The canalith repositioning procedure for treatment of BPPV. Otolaryngol Head Neck Surg 1992;107:399-404.

7 Furman JM, Cass SP. Benign paroxysmal positional vertigo. N Engl J Med 1999;341:1590-6.

8 Fetter M, Sievering F. Three-dimensional eye movement analysis in BPPV and nystagmus. Acta Otolaryngol (Stockh) 1995;115:353-7.

9 Walsh RM, Bath AP, Cullen JR, Rutka JA. Long-term results of posterior semicircular canal occlusion for intractable benign paroxysmal positioning vertigo. Clin Otolaryngol 1999;24:316-23.

10 Steddin S, Brandt T. Unilateral mimicking bilateral benign paroxysmal positioning vertigo. Arch Otolaryngol Head Neck Surg 1994;120: 1339-41.

11 Fife T. Recognition and management of horizontal canal benign positional vertigo. Am J Otol 1998;19:345-51.

12 Nuti D, Agus G, Barbieri M-T, Passali D. The management of horizontal canal paroxysmal positional vertigo. Acta Otolaryngol (Stockh) 1998; 118:455-60.

13 Andrews JC, Honrubia V. Menière's disease. In: Baloh RW, Halmagyi GM (eds), Disorders of the vestibular system. New York: Oxford University Press, 1996:300-19.

14 Gibson WPR, Arenberg IK. Pathophysiologic theories in the etiology of Menière's disease. Otolaryngol Clin North Am 1997;30:961-8.

15 Neuhauser H, Leopold M, von Brevern M, Arnold G, Lempert T. The interrelations of migraine, vertigo, and migrainous vertigo. Neurology 2001;56:436-41.

16 Bickerstaff E. Basilar artery migraine. Lancet 1961;i:15-17.

17 Baloh RW. Neurotology of migraine. Headache 1997;37:615-21.

18 Reploeg MD, Goebel JA. Migraine-associated dizziness: patient characteristics and management options. Otol Neurotol 2002; 23: 364-71.

19 Levy D. Transient CNS deficits:a common benign syndrome in young adults. Neurology 1988;38:831-6.

20 Baloh RW. Clinical practice. Vestibular neuritis. N Engl J Med. 2003; 348:1027-32.

21 Zee DS. Ophthalmoscopy in examination of patients with vestibular disorders. Ann Neurol 1978;3:373-4.

22 Halmagyi GM, Curthoys IS. A clinical sign of canal paresis. Arch Neurol 1988;45:737-9.

23 Halmagyi GM, Colebatch JG, Curthoys IS. New tests of vestibular function. Baillieres Clin Neurol 1994;3:485-500.

24 Aw ST, Fetter M, Cremer PD, Karlberg M, Halmagyi GM. Individual semicircular canal function in superior and inferior vestibular neuritis. Neurology 2001;57:768-74.

25 Amarenco P. The spectrum of cerbellar infarctions. Neurology 1991;41:973-9.

26 Thomke F, Hopf HC. Pontine lesions mimicking acute peripheral vestibulopathy. J Neurol Neurosurg Psychiatry 1999;66:340-9.

27 Rinne T, Bronstein AM, Rudge P, Gresty MA, Luxon LM. Bilateral loss of vestibular function: clinical findings in 53 patients. J Neurol 1998; 245:314-21.

28 Waterston JA, Halmagyi GM. Unilateral vestibulotoxicity due to systemic gentamicin therapy. Acta Otolaryngol (Stockh) 1998;118:474-8.
29 Halmagyi GM, Fattore CM, Curthoys IS, Wade S. Gentamicin vestibulotoxicity. Otolaryngol Head Neck Surgery 1994;111:571-4.

30 Vanneste JAL. Three decades of normal pressure hydrocephalus: are we wiser now? J Neurol Neurosurg Psychiatry 1994;57:1021-5.

31 Stolze H, Kuhtz-Buschbeck JP, Drucke H, Johnk K et al. Gait analysis in idiopathic normal pressure hydrocephalus - which parameters respond to the CSF tap test? Clin Neurophysiol 2000;111:1678-86.

32 Dunniway HM, Welling DB. Intracranial tumors mimicking benign paroxysmal positional vertigo. Otolaryngol Head Neck Surg 1998;118: 429-36.

33 Weber PC, Cass SP. Neurotologic manifestations of Chiari 1 malformation. Otolaryngol Head Neck Surg 1993;109:853-60.

34 Litvan I, Agid Y, Calne D, Campbell G et al. Clinical research criteria for the diagnosis of progressive supranuclear palsy (Steele-RichardsonOlszewski syndrome). Neurology 1996;47:1-9.

35 Schols L, Bauer P, Schmidt T, Schulte T, Riess O. Autosomal dominant cerebellar ataxias: clinical features, genetics, and pathogenesis. Lancet Neurol 2004;3:291-304

36 Shams'ili S, Grefkens J, de Leeuw B, van den Bent M et al. Paraneoplastic cerebellar degeneration associated with antineuronal antibodies: analysis of 50 patients. Brain 2003;126:1409-18.

37 Wenning GK, Kraft E, Beck R, Fowler CJ et al. Cerebellar presentation of multiple system atrophy. Mov Disord 1997;12:115-7.

38 Gerschlager W, Munchau A, Katzenschlager R, Brown P et al. Natural history and syndromic associations of orthostatic tremor: a review of 41 patients. Mov Disord 2004 July;19(7):788-95.

39 Brown P. New clinical sign for orthostatic tremor. Lancet 1995;346: 306-7.

40 Kuntzer T, Antoine JC, Steck AJ. Clinical features and pathophysiological basis of sensory neuronopathies (ganglionopathies). Muscle Nerve 2004;30:255-68

41 Minor LB. Superior canal dehiscence syndrome. Am J Otol 2000; 21:9-19.

42 Halmagyi GM, Aw ST, McGarvie LA, Todd MJ et al. Superior semicircular canal dehiscence simulating otosclerosis. J Laryngol Otol 2003; 117:553-8.

43 Neff BA, Willcox Jr TO, Sataloff RT. Intralabyrinthine schwannomas. Otol Neurotol 2003;24:299-307.

44 Bergsneider M, Becker DP. Vascular compression syndrome of the vestibular nerve: a critical analysis. Otolaryngol Head Neck Surg 1995; 112:118-24.

45 Brandt TH, Dieterich M. Vestibular parxoysmia: vascular compression of the eighth nerve? Lancet 1994;i:798-9.

46 Fisher CM. Vertigo in cerebrovascular diseases. Arch Otolaryngol Head Neck Surg 1967;85:529-34.

47 Grad A, Baloh RW. Vertigo of vascular origin. Clinical and electronystagmographic features in 84 cases. Arch Neurol 1989;46:281-4. Comment, Arch Neurol 1990;47:12-13.

48 Oas JG, Baloh RW. Vertigo and the anterior inferior cerebellar artery syndrome. Neurology 1992;42:2274-9.

49 Gomez CR, Cruz-Flores S, Malkoff MD, Sauer CM, Burch CM. Isolated vertigo as a manifestation of vertebrobasilar ischemia. Neurology 1996; 47:94-7.

50 Lee H, Yi HA, Baloh RW. Sudden bilateral simultaneous deafness with vertigo as a sole manifestation of vertebrobasilar insufficiency. J Neurol Neurosurg Psychiatry 2003;74:539-41. 